\title{
Nonlinear System Identification using Uncoupled State Multi-model Approach: Application to the PCB Soldering System
}

\author{
Sofiane Khouni \\ Department of Electrical Engineering \\ Ferhat Abbas Setif 1 University \\ Setif, Algeria \\ sofiane.khouni@cevital.com
}

\author{
Kamel Eddine Hemsas \\ Department of Electrical Engineering \\ Ferhat Abbas Setif 1 University \\ Setif, Algeria \\ hemsas_ke_dz@univ-setif.dz
}

\begin{abstract}
Multi-model approach is an adapted tool of modeling nonlinear systems. The underlying idea is to simplify the complex nature of the system to be studied by decomposing it into simple (linear) sub-systems, in order to simplify the study (stability, control law, surveillance, etc.). This technique allows us to extend the application of linear systems methodology to nonlinear systems. This paper presents nonlinear system identification using an uncoupled state multi-model applied to a Printed Circuit Boards (PCB) soldering system. Precision, simplicity, and fidelity of the obtained results show the effectiveness of the used algorithm to identify, model, and write down as simple subsystems, a complex black box system.
\end{abstract}

Keywords-nonlinear system; identification; uncoupled state; multi-model; profile modeling

\section{INTRODUCTION}

Physical systems are often complex and difficult to model. The aim of control law synthesis and/or system diagnosis is to call for a modeling phase which allows obtaining a faithful representation of the behavior of the considered system. This representation must be sufficiently accurate and simple at the same time. A nonlinear model is more suitable to offer a good representation for most real processes, compared to a linear model that remains valid only over a rather restricted area of the operating space or around a balance point. The performance of the linear model declines by moving away from it and the search for a more suitable, often nonlinear model becomes necessary. Unfortunately, the use of a nonlinear model in different contexts (identification, estimation, control, and diagnosis) becomes more difficult and more delicate than a linear model, and, on the other hand, the analysis techniques of a linear time invariant (LTI) model have been widely developed, which is not the case for nonlinear systems. The mathematical structure that can solve the problem mentioned above, while keeping the mathematical simplicity of the LTI models, is a global approach, called multi-model. The multimodel is a universal approximation approach for nonlinear systems. It allows us to extend the use of techniques adapted to linear systems on nonlinear systems. The multi-model approach is based on the idea of decomposing the complex nonlinear system into a finite number $L$ of linear sub-models $y_{i}$ valid in operating areas. The output of each sub-model $y_{i}$ is put into action through an interpolation mechanism (weighting functions) which ensures the transition from one sub-model $y_{i}$ to another. The overall behavior of the nonlinear system is thus approximated by taking into account the contribution of each sub-model. There are two types of multi-model systems, depending on the way of interconnection between the sub models, namely the coupled state multi-model or Takagi Sugeno fuzzy model [1], and the uncoupled state multi-model [2]. Nonlinear system identification using coupled state multimodel approach has been extensively studied [3-6]. On the other hand, a few works suggest an identification procedure based on an uncoupled multi-model structure [7, 8]. However, the field of electronic card (PCBs) manufacturing has recently grown significantly, due to the fact that the different areas of contemporary life require the presence of these boards in various domestic appliances. The manufacturers of these devices demand that these PCBs must be of high quality and without problems to ensure the reliability and the safety of their devices. This objective can only be achieved by ensuring the manufacturing quality of PCBs in its various phases, including soldering. Thus, soldering system monitoring in the dedicated "industrial oven" has become a necessity to ensure the quality and durability of the PCB soldering. This complex system can only be monitored after developing its mathematical model. So, in this paper, the decoupled multi-model method is used to model a real-world industrial complex system which is a soldering system of PCB into an off-line oven ("Profile" system).

\section{MULTI-MODELS}

Throughout this paper, we will designate sampled variable $v(k)$ as the value of the variable $v(t)$ at time $t=k . T_{s}$ where $T_{s}$ is the sampling period. Thus, the global output of the sampled time multi-model $y(k)$ is defined by:

$$
y(k)=\sum_{i=1}^{L} \mu_{i}(\xi(k)) y_{i}(k)
$$

where $\mu_{i}$ are the weighting functions that ensure the switching between sub-models. They are associated with each operating 
area, and are chosen to verify the convex sum properties:

$$
\begin{array}{r}
\sum_{i=1}^{L} \mu_{i}(\xi(k))=1 \text { et } 0 \leq \mu_{i}(\xi(k)) \leq 1, \\
\forall i=1 \ldots L, \quad \forall k
\end{array}
$$

$\xi(k)$ is the vector of decision or indexing variables of weighting functions $\mu_{i}$. These decision variables can be measurable state variables or input or output, or a combination of these variables, $L$ is the sub-model's number. The contribution of each sub-model $y_{i}(k)$ depends on its weighting function $\mu_{i}(\xi(k)) . \mu_{i}$ is obtained from the normalization of the activation functions $w_{i}$ for each sub-model:

$$
\mu_{i}(\xi(t))=\frac{w_{i}(\xi(t))}{\sum_{j=1}^{L} w_{j}(\xi(t))}
$$

These activation functions $w_{i}$ can be triangular, trapezoidal, Gaussian or sigmoidal.

\section{MULTI-MODEL STRUCTURES}

Depending on the coupling or interconnection nature of the local sub-models used to generate the global output of the multi-model, two large families are used: coupled state and one uncoupled state multi-models.

\section{A. Coupled State Multi-models (Takagi-Sugeno)}

Also called unique state or Takagi-Sugeno multi-models [1], they operate with sub-models sharing the same state vector $x(k)$. The sampled state representation for this multi-model is given by [9]:

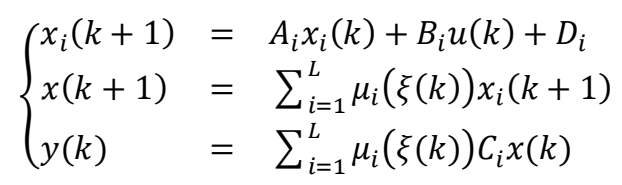

where $\mu_{i}$ satisfy the defined convexity conditions in (2), $x \in \mathbb{R}^{n}$ is the state vector, $u \in \mathbb{R}^{m}$ the input vector, $y \in \mathbb{R}^{p}$ the output vector, $\xi$ the indexation variables vector of weighting functions $\mu_{i}$.

\section{B. Uncoupled State Multi-models (Filev)}

In this type of multi-model, the sub-models are uncoupled [2], i.e. each sub-model has its own state vector $x_{i}(k)$, so the dimensions and complexity of the sub-models vary. The sampled state representation for this multi-model is given by $[8,10]$ :

$$
\begin{cases}x_{i}(k+1) & =A_{i} x_{i}(k)+B_{i} u(k)+D_{i} \\ y_{i}(k) & =C_{i} x_{i}(k) \\ y(k) & =\sum_{i=1}^{L} \mu_{i}(\xi(k)) y_{i}(k)\end{cases}
$$

where $\mu_{i}$ satisfy the defined convexity conditions in (2), $x_{i} \in \mathbb{R}^{n_{i}}$ is the state vector of the $i^{\text {th }}$ sub-model, $u \in \mathbb{R}^{m}$ is the input vector, $y \in \mathbb{R}^{p}$ the output vector, $\xi$ the indexation variables vector of multi-model obtaining methods. It is suitable to note that the outputs $y_{i}(k)$ of the sub-models are "artificial modeling signals" used only to describe the nonlinear behavior of the real system. These signals, inaccessible to measurement and devoid of any physical sense, are connected to the real system only by their weighted sum [8].

\section{MULTI-MODEL OBTAINING METHODS}

A nonlinear system is given by the following general state and input-output relations:

$$
\left\{\begin{array}{l}
x(k+1)=f(x(k), u(k)) \\
y(k)=g(x(k), u(k))
\end{array}\right.
$$

The objective of multi-model modeling is to simplify the complex nonlinear functions $f($.$) and g($.$) . On the other hand,$ modeling a nonlinear system by a multi-model amounts to defining these parameters, i.e. $A_{i}, B_{i}, C_{i}$ and $D_{i}$ with parameters of the weighting functions of the sub-models in (4) and (5). Three well-known methods to obtain these parameters are:

- Linearization around operating points $[9,11,12]$.

- Transformation by nonlinear sectors $[11,13]$.

- Parametric identification $[5,7,8,14]$.

\section{PARAMETRIC IDENTIFICATION OF UNCOUPLED STATE MULTI-MODEL}

The identification of a multi-model concerns the search of an optimal structure and the estimation of the parameters of the weighting functions and of the local models. This method can be applicable if only the measurements of the inputs and outputs of the system (with no mathematical model or black box) are available [5], by searching or imposing the structure of the multi-model that is the input/output behavior, without interest in obtaining a knowledge model who describes its internal behavior (phenomenological), the global representation of the identification method is illustrated in Figure 1.

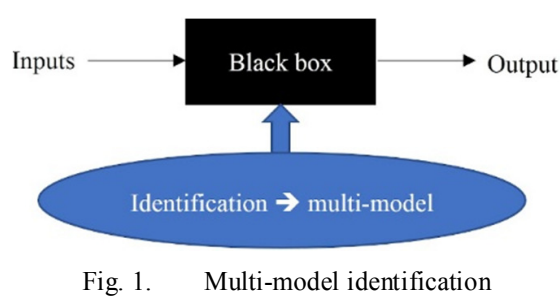

There are two steps in obtaining a multi-model identification:

- Determination of the structure $\rightarrow$ Partitioning of the inputoutput data which leads to: determining the number of local models, choosing the decision variables, and realizing the partitioning of the space and determining the parameters of the weighting functions.

- Identifying sub-model parameters using an iterative approach.

\section{A. Problem Formulation}

A sampled time uncoupled multi-model and function of an unknown parameter vector $\theta$, is in the form:

$$
\begin{cases}x_{i}(k+1) & =A_{i}\left(\boldsymbol{\theta}_{i}\right) x_{i}\left(k, \boldsymbol{\theta}_{i}\right)+B_{i}\left(\boldsymbol{\theta}_{i}\right) u(k)+D_{i}\left(\boldsymbol{\theta}_{\boldsymbol{i}}\right) \\ y_{i}(k) & =C_{i}\left(\boldsymbol{\theta}_{\boldsymbol{i}}\right) x_{i}\left(k, \boldsymbol{\theta}_{\boldsymbol{i}}\right) \\ y(k) & =\sum_{i=1}^{L} \mu_{i}(\xi(k)) y_{i}\left(k, \boldsymbol{\theta}_{\boldsymbol{i}}\right)\end{cases}
$$

where: 
$\boldsymbol{\theta}_{\boldsymbol{i}}$ : Are the parameters of the sub-model $i$ in the form of a column vector of dimension $q_{i}$. For the purposes of the identification procedure, all parameters of the sub-models are grouped together in a single augmented column vector $\theta$, where:

$$
\begin{aligned}
& \theta=\underbrace{\left[\begin{array}{llllll}
\theta_{1} & \theta_{2} & \cdots & \theta_{i} & \cdots & \theta_{L}
\end{array}\right]^{T}}_{\text {components number }=\sum q_{i}} \\
& \theta_{i}=\left[\begin{array}{lllll}
\theta_{i, 1} & \ldots & \theta_{i, q} & \ldots & \theta_{i, q_{i}}
\end{array}\right]^{T}
\end{aligned}
$$

$q_{i}$ : Stands for the parameter's number in sub-model $i$.

$\theta_{i, q}$ : Is the $q^{\text {th }}$ parameter in sub-model $i$, it is a scalar to identify.

Thus, the problem of identification is summarized as follows: the weighting functions $\mu_{i}($.$) are fixed, and from the$ input $u(t)$ and the measured output $y_{s}(t)$ (of a MISO or SISO system), $\theta$ is the parameter vector of the multi-model must be identified.

\section{B. Implementation of Identification}

The sub-model parameter identification is done by minimizing a criterion which presents the difference between the measured output (original system) and the estimated output (the multi-model). Most often the criterion used is of a quadratic form:

$$
\begin{aligned}
& J_{G}(\theta)=\frac{1}{2} \sum_{k=1}^{N} \varepsilon^{2}(k, \theta) \\
& \varepsilon(k, \theta)=y(k, \theta)-y_{s}(k)
\end{aligned}
$$

where $y(k)$ is the multi-model output, $y_{s}(k)$ the measured output of the nonlinear system and $N$ is the measurement number.

The criterion $J_{G}$ in (9) is a global one. It is used if we want to obtain a behavioral multi-model in a global way. It is also possible to use a local criterion if we want to obtain a multimodel that favours a good match between the local behavior of sub-models and of the nonlinear system in each operating zone. The local criterion is given by:

$$
J_{L}(\theta)=\sum_{i=1}^{L} \sum_{k=1}^{N} \mu_{i}(\xi(k))\left(y_{i}(k, \theta)-y_{s}(k)\right)^{2}
$$

where $y_{i}(k)$ is the $i^{\text {th }}$ sub-model output.

It is also possible to combine two criterions in a single mixed criterion of the form:

$$
J_{M}(\theta)=\alpha J_{G}+(1-\alpha) J_{L}, \quad 0 \leq \alpha \leq 1
$$

The parametric estimation procedure of the parameter vector $\theta$ is done iteratively, with each iteration approximating the optimal solution of the vector $\theta$. This procedure is implemented by using the Gauss-Newton algorithm. The updating of the parameter vector at each iteration $n$ is obtained from the following recurring formula:

$$
\theta(n+1)=\theta(n)-H(\theta)^{-1} G(\theta)
$$

where $\theta(n)$ is the parameter vector at iteration $n, \theta(n+1)$ the same vector at the following iteration, $H(\theta)=\frac{\partial^{2} J(\theta)}{\partial \theta \partial \theta^{T}}$ is the hessian matrix, $G(\theta)=\frac{\partial J(\theta)}{\partial \theta}$ is the gradient vector, and $J$ is a global, local, or mixed criterion. The computation of the gradient vector $G$ and the hessian matrix $H$ is based on the computation of the sensitivity functions of the multi-model output with respect to the parameters of each sub-model. In order to ensure and improve the convergence of (12) the following Marquardt formula is recommended [15]:

$$
\theta(n+1)=\theta(n)-\Delta(n)(H(\theta)+\beta(n) I)^{-1} G(\theta)(13)
$$

where: $\Delta(n)$ : the relaxation coefficient of the iteration $n$, with $\Delta(n) \leq 1, \forall n$, and $\beta(n)$ : the regularisation parameter, $\beta(1)$ is chosen near zero, i.e.: $\beta(1) \approx 0$.

\section{1) Computation of Gradient Vector $G_{G}$}

The gradient vector $G_{G}$ is calculated by derivation of the global criterion in (9) with respect to the parameters vector:

$$
\begin{gathered}
G(\theta)=\frac{\partial J_{G}(\theta)}{\partial \theta}=\frac{\partial\left(\frac{1}{2} \sum_{k=1}^{N} \varepsilon^{2}(k, \theta)\right)}{\partial \theta} \\
G(\theta)=\sum_{k=1}^{N} \varepsilon(k, \theta) \frac{\partial y(k, \theta)}{\partial \theta}
\end{gathered}
$$

with :

$$
\frac{\partial y(k, \theta)}{\partial \theta}=\sum_{i=1}^{L} \mu_{i}(\xi(k)) \frac{\partial y_{i}(k, \theta)}{\partial \theta}
$$

The term $\frac{\partial y_{i}(k, \theta)}{\partial \theta}$ represents the sensitivity functions of the $1^{\text {st }}$ order of the $i^{\text {th }}$ output with respect to the parameter vector $\theta$ of the multi-model.

\section{2) Computation of Hessian Matrix $H_{G}$}

It is obtained directly from the gradient by derivation with respect to the parameter vector:

$$
\begin{gathered}
H_{G}(\theta)=\frac{\partial^{2} J_{G}(\theta)}{\partial \theta \partial \theta^{T}} G(\theta) \\
H_{G}(\theta)=\sum_{k=1}^{N} \underbrace{\varepsilon(k)}_{\rightarrow 0} \frac{\partial^{2} y(k, \theta)}{\partial \theta \partial \theta^{T}}+\sum_{k=1}^{N} \frac{\partial y(k, \theta)}{\partial \theta} \frac{\partial y(k, \theta)}{\partial \theta^{T}}(16)
\end{gathered}
$$

$\varepsilon(k)$ is negligible under the assumption that the error tends to zero, in this case the hessian matrix becomes:

$$
H_{G}(\theta)=\frac{\partial^{2} J_{G}(\theta)}{\partial \theta \partial \theta^{T}} \approx \sum_{k=1}^{N} \frac{\partial y(k, \theta)}{\partial \theta} \frac{\partial y(k, \theta)}{\partial \theta^{T}}
$$

3) Computation of Sensitivity Functions

$$
\frac{\partial y_{i}(k, \theta)}{\partial \theta_{p, q}}=\frac{\partial C_{i}(\theta)}{\partial \theta_{p, q}} x_{i}(k, \theta)+C_{i}(\theta) \frac{\partial x_{i}(k, \theta)}{\partial \theta_{p, q}}
$$

where:

$i$ : is the index of the $i^{\text {th }}$ sub-model,

$p$ : the index of the $p^{\text {th }}$ parameter vector of the $p^{\text {th }}$ sub-model, $q$ : the $q^{\text {th }}$ parameter in $\theta_{p}$ vector, and:

$$
\begin{aligned}
\frac{\partial x_{i}(k, \theta)}{\partial \theta_{p, q}}= & \frac{\partial A_{i}(\theta)}{\partial \theta_{p, q}} x_{i}(k, \theta)+A_{i}(\theta) \frac{\partial x_{i}(k, \theta)}{\partial \theta_{p, q}} \\
& +\frac{\partial B_{i}(\theta)}{\partial \theta_{p, q}} u(k)+\frac{\partial D_{i}(\theta)}{\partial \theta_{p, q}}, k=2,3, \ldots
\end{aligned}
$$




\section{4) Parametric Identification Algorithm for a Multi-model}

Figure 2 summarizes the steps constituting the algorithm of the parametric identification described above.

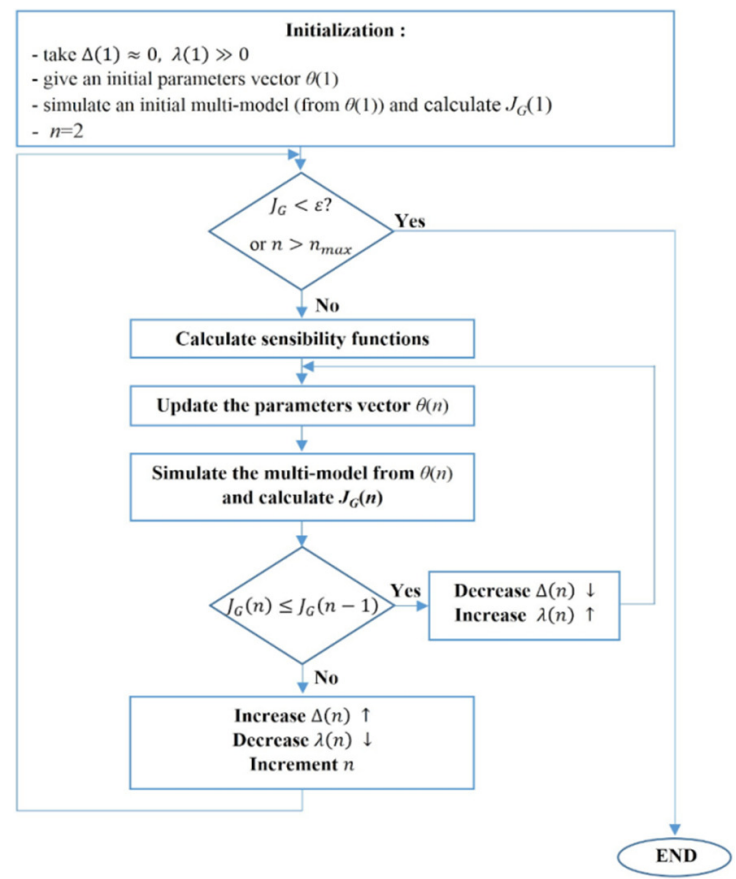

Fig. 2. Parametric identification algorithm for a multi-model

\section{APPLICATION}

\section{A. System Description}

\section{1) SMT Process}

SMT (Surface Mounted Technology) means: surface mounted technology of the electronic components on the PCB, in other words, non through the whole component but placed on the surface of the PCB. This process is carried out within a production line of PCB. The placement of the SMD (Surface Mounted Device) is done by special machines called pick and place machines who place the SMD on blank boards. The PCB and SMD will be soldered automatically by passing through an industrial in-line reflow dedicated oven. The high heat inside the reflow oven melts the alloys contained in the paste to weld the components already mounted on the PCB.

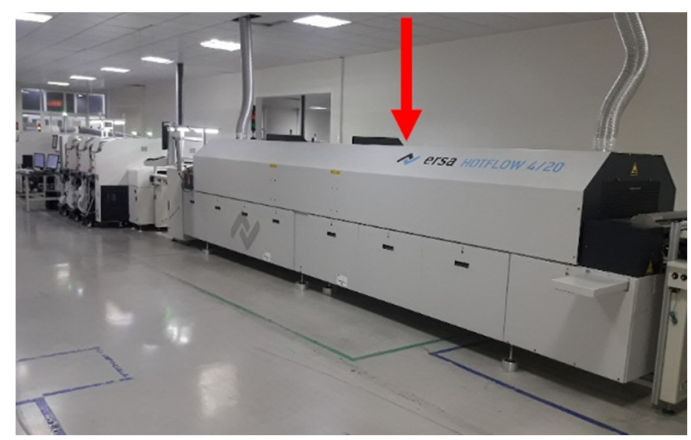

Fig. 3. In-line industrial reflow oven (dual-lane $14 \times 2$ zones) in SMT line
This operation, (soldering board in a reflow oven), is divided into 4 steps:

- Preheating: Increases the temperature of the components and solder paste.

- Drying: Time for the flux to act and evaporate completely.

- Reflow: Fusion of the solder paste and rise to the maximum temperature which must be the melting temperature.

\section{- Cooling.}

The variation of the temperature is studied and regulated very strictly so that the soldering is of good quality.

\section{2) Profile}

A "Profile" is a graph that represents the temperature of the PCB soldering in the reflow oven, i.e. represents the variation of the temperature as a function of time $T^{\circ}(t)$ during this operation. This function depends mainly on the solder paste and the product to be manufactured. The variation of the temperature in a Profile passes through the four steps mentioned above: preheating, drying, reflow and cooling. The general form of the Profile is illustrated in Figure 4.

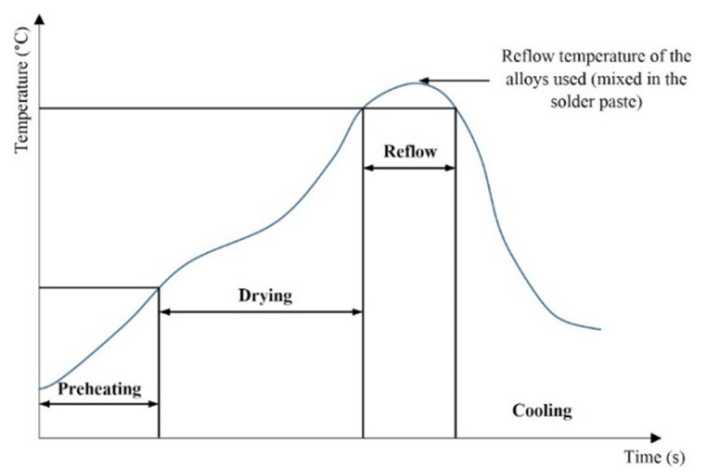

Fig. 4. General form of the Profile

\section{3) System Description: "Profile of Forced Convection Oven HYSC CO-81"}

This type of oven (Figure 5) belongs to the family of desktop reflow ovens or single-zone off-line reflow ovens. It can be used off-line, and has the advantage of being simple (mono-zone) as opposed to the multi-zone reflow oven (Figure 3 ), which makes its modeling possible in the context of multimodels. This oven is composed of a single phase thermoelectric actuator (heating resistor), an SSR (Solid Static Relay) which supplies the electrical power to the thermoelectric actuator, and an electronic controller that regulates the power of the SSR. The most important specifications of this oven are [16]:

- Temperature: $\max =250^{\circ} \mathrm{C}$.

- Controller: Digital PID controller.

- Heater: Sheath heater.

- Heating : Convection (forced by fans). 
- Electric Requirements: 220VAC, 50/60Hz.

- Power consumption: 1600W.



Fig. 5. Industrial forced convection oven HYSC CO-81

The profile of this oven can be considered as an automated SISO (Single In-Single Out) system, the input is the average power supplied by the SSR (power transformer), and the output is the Profile itself, as shown in Figure 6.

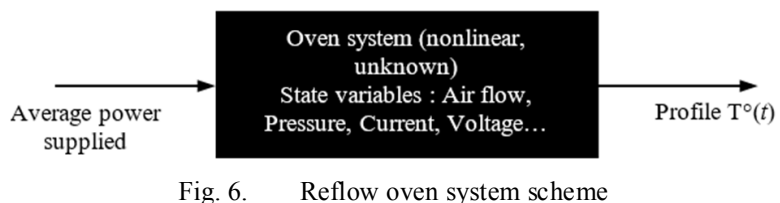

Heat (energy) generated by the heating resistor and produced by the electrical power, is propagated by the phenomenon of forced convection by fans. The temperature in this case (the profile) is that of the paste solder measured by a temperature probe installed in a special device called Profiler, which is connected to the PC for data collection $\left(\mathrm{T}^{\circ}(t)\right)$. The formula that relates the average power dissipated in the heaters over a period $T_{c}$ (cycle time of the SSR) and the cyclic rate $\alpha$ is:

$$
P_{\text {avg }}=\alpha \frac{V_{\text {eff }}^{2}}{R}
$$

where $R$ is the resistance.

The input $P_{\text {avg }}$ in one PCB soldering cycle is represented in Figure 7.

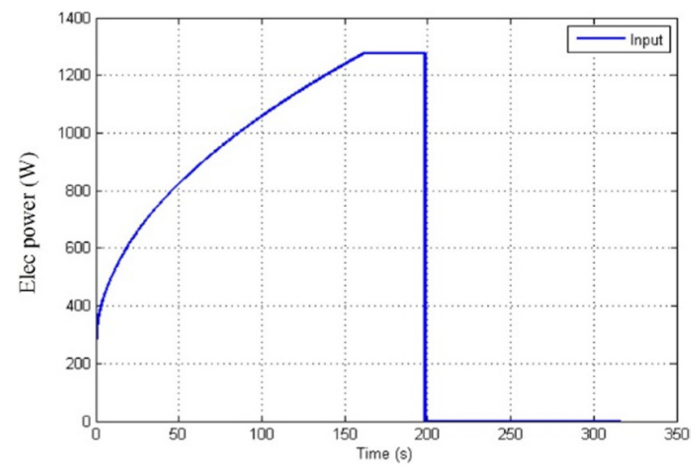

Fig. 7. System input (average power)
Note that the PCB soldering cycle for this measurement is $315 \mathrm{~s}$. The measured output $y_{s}(t)$ or the profile is represented by Figure 8 (in the same soldering cycle).

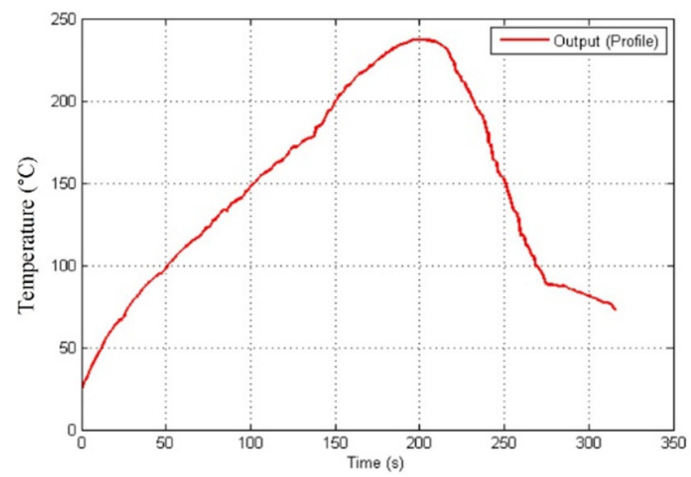

Fig. 8. System output (profile)

The general formula linking the input $u(t)=P_{\text {moy }}(t)$, the state vector $X(t)$ and the measured output $y_{s}(t)=T^{\circ}(t)$ of this system is:

$$
y_{S}(t)=g(X, u)
$$

Note that the nonlinear function $g$ is unknown, so we are facing an identification problem.

\section{B. Multi-model Modeling}

Since there is no mathematical relation or equation linking the input and the output, we will use identification to obtain the parameters of the sub-models. The first step is the partitioning of the operating space and the choice of the parameters of the weighting functions. For simplifying reasons, it has been assumed that the partitioning and the parameters of the weighting functions are known. The operating space is divided into five zones, which means that we would consider five submodels and five weighting functions. The activation functions used for the normalization of the weighting functions are chosen to be Gaussian. Note that this partitioning is not unique. The decision variable is the measured output of the system, $\xi(t)=y_{s}(t)$. Each activation function $w_{i}$ of a sub-model $i$, of centre $c_{i}$ and of dispersion coefficient $\sigma$ (which is assumed to be common for all sub-models) is of the following form:

$$
w_{i}(\xi(t))=e^{-\left(\frac{\xi(t)-c_{i}}{\sigma}\right)^{2}}
$$

The weighting function $\mu_{i}(\xi(t))$ of a sub-model $i$ is given by Figure 9 , in which we see that the weighting functions are highly mixed, this choice is appropriate for the global parametric estimation method. The degrees of the five submodels are respectively: $1,1,2,2,1$. This arbitrary choice is based on the form of the measured output $y_{s}(t)$, which depends on the complexity of the five operating areas. Hence, the form of the five sub-models is:

$$
\begin{aligned}
& \begin{cases}x_{i}(k+1) & =A_{i}\left(\boldsymbol{\theta}_{\boldsymbol{i}}\right) x_{i}(k)+B_{i}\left(\boldsymbol{\theta}_{\boldsymbol{i}}\right) u(k)+D_{i}\left(\boldsymbol{\theta}_{\boldsymbol{i}}\right) \\
y_{i}(k) & =C_{i}\left(\boldsymbol{\theta}_{\boldsymbol{i}}\right) x_{i}(k)\end{cases} \\
& i=1 . .5
\end{aligned}
$$


where: $A_{i}=a_{i}, B_{i}=b_{i}, D_{i}=d_{i}, C_{i}=c_{i}, a_{i}, b_{i}, d_{i}, c_{i}$ are scalars, and $\boldsymbol{\theta}_{i}=\left[\begin{array}{llll}a_{i} & b_{i} & d_{i} & c_{i}\end{array}\right]^{T}$ for $i=1,2$ and 5 , $a_{i, 1 . .4}, b_{i, 1.2}, d_{i, 1.2}, c_{i, 1.2}$ are scalars, and

$\boldsymbol{\theta}_{\boldsymbol{i}}=\left[\begin{array}{llllllllll}a_{i, 1} & a_{i, 2} & a_{i, 3} & a_{i, 4} & b_{i, 1} & b_{i, 2} & d_{i, 1} & d_{i, 2} & c_{i, 1} & c_{i, 2}\end{array}\right]^{\boldsymbol{T}}$

for $i=3$ and 4 .

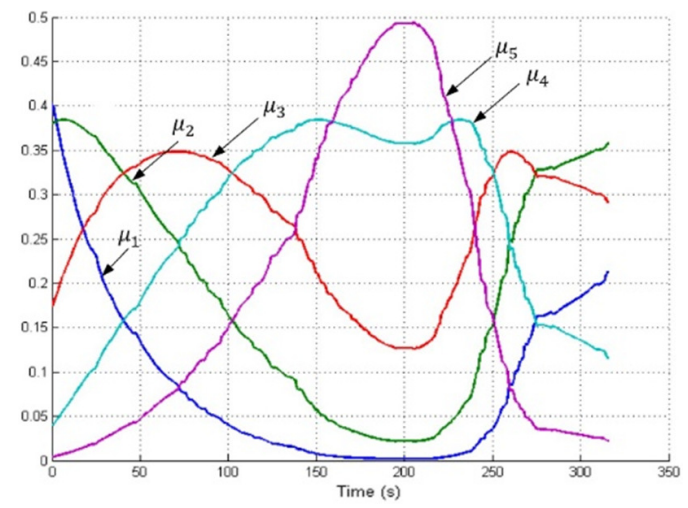

Fig. 9. Weighting functions (highly mixed)

The application of the identification algorithm gives us the following results, with $S_{1 \ldots 5}$ being sub-model parameters:

$$
\begin{aligned}
& S_{1}:\left\{\begin{array}{l}
A_{1}=a_{1}=0.0283 ; \quad B_{1}=b_{1}=0.0496 \\
D_{1}=d_{1}=0.1469 ; C_{1}=c_{1}=1.0102
\end{array}\right\} \\
& S_{2}:\left\{\begin{array}{l}
A_{2}=a_{2}=0.0843 ; \quad B_{2}=b_{2}=-0.0228 \\
D_{2}=d_{2}=0.3911 ; C_{2}=c_{2}=1.0637
\end{array}\right\} \\
& S_{3}:\left\{\begin{array}{l}
A_{3}=\left[\begin{array}{ll}
0.4707 & 0.5291 \\
0.4501 & 0.5300
\end{array}\right] ; B_{3}=\left[\begin{array}{c}
0.1536 \\
-0.1334
\end{array}\right] \\
D_{3}=\left[\begin{array}{ll}
0.7299 \\
0.7297
\end{array}\right] ; C_{3}=c_{3}=\left[\begin{array}{ll}
1.3740 & 1.2510
\end{array}\right]
\end{array}\right\} \\
& S_{4}:\left\{\begin{array}{l}
A_{4}=\left[\begin{array}{cc}
0.9863 & 0.0928 \\
0.0021 & 0.0084
\end{array}\right] ; B_{4}=\left[\begin{array}{c}
-0.0068 \\
0.0701
\end{array}\right] \\
D_{4}=\left[\begin{array}{c}
1.0738 \\
-0.0000
\end{array}\right] ; C_{4}=c_{4}=\left[\begin{array}{ll}
1.5572 & -0.0340
\end{array}\right]
\end{array}\right\} \\
& S_{5}:\left\{\begin{array}{l}
A_{5}=a_{5}=0.9967 ; \quad B_{5}=b_{5}=0.0001 \\
D_{5}=d_{5}=1.0641 ; C_{5}=c_{5}=1.5994
\end{array}\right\}
\end{aligned}
$$

The five sub-models $y_{i}$ are exhibited in Figure 10.
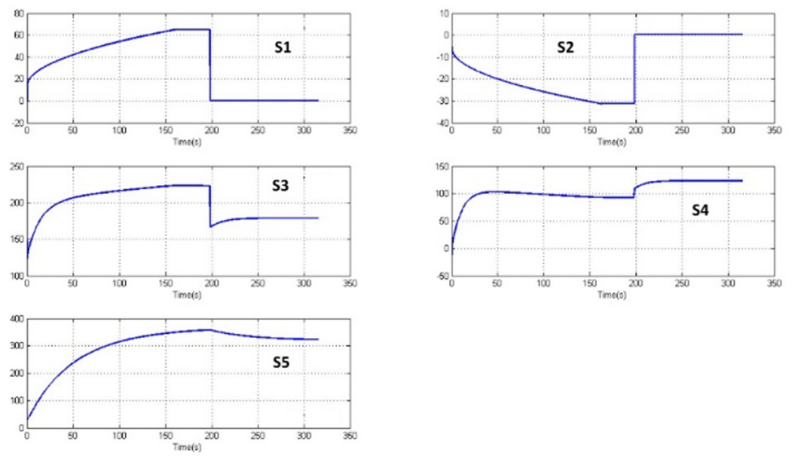

Fig. 10. The five sub-models $y_{i}$

The global multi-model output is represented in Figure 11.

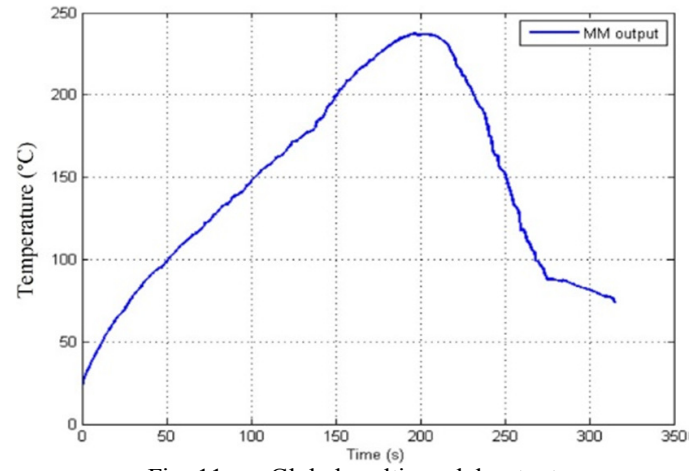

Fig. 11. Global multi-model output

\section{Multi-model Precision}

The precision of a multi-model is described by two performance indices, the first being the MSE (Mean Square Error) given by:

$$
M S E=\frac{1}{N} \sum_{k=1}^{N}\left(y_{S}(k)-y(k)\right)^{2}
$$

The second is the VAF (Variance Accounted For) given in percentage. The evaluation of the VAF allows, in the general case, to compare two matrices. The VAF is equal to $100 \%$ if the two matrices $M$ and $\widehat{M}$ are identical. Otherwise, the VAF varies between $0 \%$ and $100 \%$. It is defined by:

$$
V A F=\max \left(1-\frac{\operatorname{var}(M-\widehat{M})}{\operatorname{var}(\widehat{M})}, 0\right) \times 100 \%
$$

Where $M$ is the measured data matrix and $\widehat{M}$ the estimated data matrix (the multi-model in our case). In the obtained multimodel, $\mathrm{MSE}=0.0727$, and $\mathrm{VAF}=99.998 \%$. Figure 12 gathers the measured output (Profile) and the estimated output (multimodel) in the same view, allowing the precision of the applied multi-model identification to be shown.

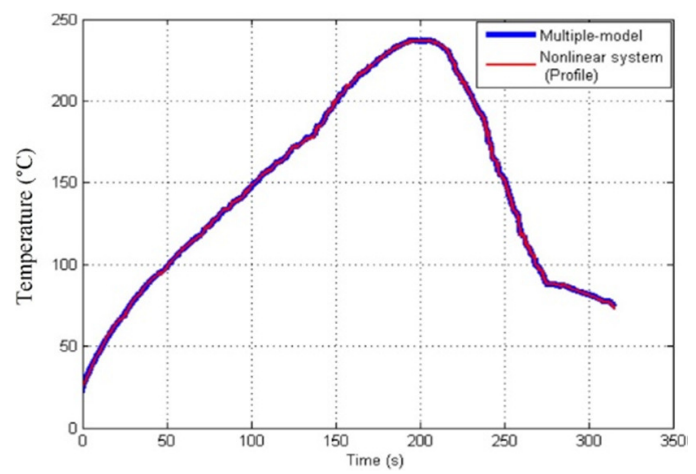

Fig. 12. The multi-model identification precision

\section{CONCLUSION}

The multi-model is a high precision global approximation approach for nonlinear systems. Its principle is "divide and rule", i.e. it eliminates the complexity of a nonlinear system by decomposing it into a finite number of simple (linear) subsystems that are easy to manipulate. The output of each submodel contributes through an interpolation mechanism (weighting functions) which ensures the transition from one sub-model to another. The overall behavior of the nonlinear 
system is thus approximated by taking into account the contribution of each sub-model. The major advantage of this approach is to extend the application of the methods developed for linear systems to nonlinear systems. Depending on the nature of the coupling of the local sub-models associated with the different zones of operation, two large multi-model families are utilized, global state multi-models whose sub-models share the same operating space and uncoupled state multi-models where each sub-model has its own state vector. To obtain these multi-models, three main methods are noted: linearization around operating points, transformation into nonlinear sectors, and parametric identification, which has been presented in detail in this paper for uncoupled state multi-model. Despite its difficulty in terms of identification, this type of multi-model has the advantage of being simple to study in particular for monitoring and diagnosis. The uncoupled state multi-model identification method was applied to an off-line PCB soldering system profile. The obtained precision was described by $\mathrm{MSE}=0.0727$ and $\mathrm{VAF}=99.998 \%$. These two performance indices clearly show the efficiency and the accuracy of this method. The profile of an off-line and single-zone oven (Figure 5 ) was modeled as a single nonlinear system. In the case of online ovens (Figure 3) of several heating and cooling zones, which are used for all production lines because of their reliability and capacity (up to $15 \mathrm{PCB}$ s at the same time every 3 minutes), the profile is more complicated and a factor of several parameters and variables. In this case it can be considered as an automated switching system or HDS (Hybrid Dynamical System), where each mode is modeled by a multimodel.

\section{REFERENCES}

[1] T. Takagi, M. Sugeno, "Fuzzy identification of systems and its applications to modelling and control", IEEE Transactions On Systems, Man and Cybernetics, Vol. 15, No. 1, pp. 116-132, 1985

[2] D. Filev, "Fuzzy modeling of complex systems", International Journal of Approximate Reasoning, Vol. 5 No. 3, pp. 281-290, 1991

[3] T. A. Johansen, B. A. Foss, "Non linear local model representation for adaptive systems", Singapore International Conference on Intelligent Control and Instrumentation, Singapore, February 17-21, 1992

[4] T. A. Johansen, B. A. Foss, "Constructing NARMAX using ARMAX", International Journal of Control, Vol. 58, No. 5, pp. 1125-1153, 1993

[5] K. Gasso, Identification des systemes dynamiques non-lineaires: approche multi-modele, $\mathrm{PhD}$ Thesis, National Polytechnic Institute of Lorraine, 2000 (in French)

[6] J. K. Gugaliya, R. D. Gudi, S. Lakshminarayanan, "Multi-model decomposition of nonlinear dynamics using a fuzzy-CART approach", Journal of Process Control, Vol. 15, No. 4, pp. 417-434, 2005

[7] H. Elaggoune, M. Benouaret, M. Messaadia, "Modeling and fault diagnosis sensor by multi-model approach", 10th International Confernece on Conception and Integrated Production, Tanger, Morroco, December 2-4, 2015

[8] R. Orjuela, B. Marx, J. Ragot, D. Maquin, "Nonlinear system identification using heterogeneous multiple models", International Journal of Applied Mathematics and Computer Science, Vol. 23, No. 1, pp. 103-105, 2013

[9] Z. Lendek, T. M. Guerra, R. Babuska, B. De Schutter, Stability analysis and nonlinear observer design using Takagi-Sugeno fuzzy models, Springer-Verlag, 2010

[10] H. Bedoui, A. Kedher, K. Ben Othman, "Fault detection and isolation for an uncertain Takagi-Sugeno fuzzy system using the interval approach", in: Handbook of Research on Advanced Intelligent Control Engineering and Automation. IGI Global, 2015
[11] K. Tanaka, H. O. Wang, Fuzzy control systems design and analysis: A linear matrix inequality approach, John Wiley \& Sons, 2001

[12] A. Akhenak, Conception d'observateurs non lin'eaires par approche multimod'ele: application au diagnostic, $\mathrm{PhD}$ Thesis, National Polytechnic Institute of Lorraine, 2004 (in French)

[13] M. Chadli, P. Borne, Multiple models approach in automation: TakagiSugeno fuzzy systems, John Wiley \& Sons, 2012

[14] R. Isermann, M. Munchhof, Identification of dynamic systems: an introduction with applications, Springer-Verlag, 2011

[15] D. W. Marquardt, "An algorithm for least-squares estimation of nonlinear parameters", Journal of the Society for Industrial and Applied Mathematics, Vol. 11, No. 2, pp. 431-441, 1963

[16] http://www.hysc.co.kr/wp/item/forced-convection-oven/\#tab-id-2 